\title{
Procedimiento para el análisis de los primeros pasos en carrera de los velocistas
}

\author{
Alberto Miguel Morales-Fábrega \\ amfabregas@uclv.cu \\ Universidad "Marta Abreu” Villa Clara-Cuba \\ Alberto Bautista Sánchez-Oms \\ asoms@uclv.cu \\ Universidad "Marta Abreu" Villa Clara-Cuba \\ Antonio Jesús Pérez-Sierra \\ antonio.perez@unison.mx \\ Universidad de Sonora-México \\ Julio Montes-Brown \\ julio.montes@unison.mx \\ Universidad de Sonora-México \\ Jorge Luís Sánchez-Jimenez \\ jlsanchez2811@gmail.com \\ Instituto de Cultura Física y el Deporte, Sinaloa- México \\ Alfredo Portela-Saenz \\ aportela052@gmail.com \\ Universidad de Sonora-México
}

\section{RESUMEN}

La presente investigación surge como demanda tecnológica por las deficiencias técnicas en la ejecución de la fase inicial de aceleración, específicamente en los cuatro primeros pasos en las carreras de velocidad manifestadas por los atletas juveniles de la Escuela de Iniciación Deportiva Escolar de Villa Clara, Cuba; mediante el diagnóstico de las deficiencias técnicas se determinaron las variables cinemáticas a estudiar, en aras del perfeccionamiento técnico de esta fase. Los objetivos son establecer procedimientos biomecánicos para el análisis de los primeros pasos de carrera de los velocistas juveniles. Conocer el nivel de satisfacción de los usuarios con el procedimiento propuesto. Se emplearon métodos del nivel teórico y empírico; además del programa biomecánico Kinovea para obtener los grados de los diferentes partes del cuerpo, nos permitió constar los deficientes resultados que experimenta esta disciplina a nivel nacional y de la 
necesidad de contar con un procedimiento biomecánico que orienten el proceso de entrenamiento de los velocistas juveniles. Como principal resultado se obtuvo un procedimiento biomecánico para el análisis de los primeros pasos de las carreras de velocidad, donde los usuarios están muy satisfechos.

Palabras clave: procedimiento; velocistas; pasos. 


\title{
Procedure for the analysis of the first running steps of sprinters
}

\begin{abstract}
The present research arises as a technological demand due to the technical deficiencies in the execution of the initial phase of acceleration, specifically in the first four steps in sprint races manifested by the juvenile athletes of the School of School Sports Initiation of Villa Clara, Cuba; through the diagnosis of the technical deficiencies, the kinematic variables to be studied were determined, for the sake of the technical improvement of this phase. The objectives are to establish biomechanical procedures for the analysis of the first running steps of juvenile sprinters. To know the level of satisfaction of the users with the proposed procedure. Theoretical and empirical methods were used; in addition to the biomechanical program Kinovea to obtain the degrees of the different parts of the body, it allowed us to note the deficient results experienced by this discipline at national level and the need to have a biomechanical procedure to guide the training process of juvenile sprinters. As a main result, we obtained a biomechanical procedure for the analysis of the first steps of sprint races, where the users are very satisfied.
\end{abstract}

Keywords: procedure; sprinters; steps.

Artículo recibido: 05 octubre. 2021 Aceptado para publicación: 02 noviembre 2021 Correspondencia: antonio.perez@unison.mx Conflictos de Interés: Ninguna que declarar 


\section{INTRODUCCIÓN}

Uno de los deportes más investigado por las ciencias aplicadas a la Cultura Física y el Deportes, lo constituye el atletismo, en especial los sprinters, o las carreras de velocidad de 100 metros planos.

Investigaciones llevadas a cabo en el campo de la Biomecánica como (Comín, et al., 1996; Rius, et al., 2006; Carrillo, 2014; Čoh, et al., 2017; Estrada, 2018), esta ciencia estudia la estructura dinámica y cinemática de los movimientos humanos; además de analizar y determinar los factores de los sprinters, o las carreras de velocidad, ha sido significativa como referencia para el procedimiento para los primeros pasos de carrera en los velocistas.

Al respecto, Burruel et al. (2016), realiza un estudio del análisis cinemático, desde la salida de velocidad o la salida de tacos, en la primera fase, que se lleva a cabo carrera en la velocidad desde $1928-1929$.

La salida de los bloques de arrancada, constituyen parte imprescindible para realizar de manera efectiva y eficiente el desplazamiento de la primera fase de la carrera, aún más cuando se acentúan determinante para la reacción y rapidez de las acciones motoras, debido a que una salida inadecuada condiciona la aceleración resultante del atleta y por tanto una adquisición de la velocidad máxima más tardía. (Bezodis, 2009, citado en Burruel, et al., 2016, p.1)

Por tal motivo, coincidimos con Mackala (2007), realizo el estudio de las distintas fases de la carrera (aceleración, máxima velocidad y deceleración) la velocidad, amplitud y frecuencia de un grupo de ocho velocistas de alto nivel a lo largo de una prueba de 100 $\mathrm{m}$ lisos, con el propósito de comprobar de distintos factores o variables que influyen en las fases de la carrera para una mejor eficiencia de la técnica como rendimiento de la formación de hábitos y habilidades para brindar elementos analíticos de los niveles movimientos motrices y control, deben ser ejecutados por los atletas durante el proceso de su intervención durante el proceso de los gestos técnicos.

Es importante resaltar, el estudio y análisis biomecánico, desde una perspectiva cualitativa, donde el método de examen del movimiento humano es indispensable para la aproximación sistemática (Knudson \& Morris, 2002).

Estos aspectos biomecánicos deben ser atendidos por el entrenador durante el proceso de formación del entrenamiento deportivo en los velocistas, para ello es preciso el empleo 
de la biomecánica, para detectar y hacer más simple la observación y poder capturar toda la información que brindan los movimientos para caracterizar al velocista desde indicadores como: longitud del paso, frecuencia de los pasos, altura del centro de maza, nivel que alcanza la pierna en el paso anterior, ángulo del despegue posterior y del apoyo anterior entre otros.

Por esta razón compartimos la idea de los resultados obtenidos, relacionada con la salida de los tacos de manera eficiente como factor o variable eficiente es uno de los factores más importantes para tener en cuenta el estudio del procedimiento para el análisis de los primeros pasos de carrera de los velocistas, según (Ĉoh \& Tomažin, 2006).

Es importante resaltar, los estudios biomecánicos realizados por el Padullés (1992) donde plantea que:

A partir del análisis del movimiento de los seres vivos y los procedimientos para los primeros pasos de carrera de los velocistas, considerada desde varias ópticas de compresión como:

Estructural: Del cuerpo como sistema estructural complejo, constituido por esqueleto, músculos, tendones.

Dirección o regulación: Donde parte del sistema nervioso central, y los sistemas que los conforman (sistema nervioso, el sistema periférico y, sistema vegetativo) del control y regulación de los movimientos.

Mecánico: En este aspecto debemos tener presente las capacidades condicionales y coordinativas, en especial la fuerza (interna y externa).

Cibernético: La aplicación en sistemas de simulación en ordenador o robots.

Sistema complejo: Basado en las teorías del caos que pretende explicar los movimientos a partir de las aplicaciones de dinámica de sistemas. (p. 2).

Los especialistas cubanos de las pruebas de velocidad tan han realizados aportes en las últimas décadas para el deporte cubano. Donde plantea que impresionante, los corredores que han realizado hazañas inolvidables que muy pocos países pueden en las competencias de alto nivel lograr altos resultados deportivos en las pruebas de velocidad; además planeta que estos resultados obtenidos son dados por investigaciones con el programa software Kinovea (Muñiz, 2018).

La Biomecánica como ciencia aplicada a la Cultura Física tiene un papel fundamental en el estudio de la concepción científica-metodológica para el análisis de estudio del 
procedimiento de los primeros pasos de carrera en los velocistas, donde el gesto técnico como objeto de estudio, tienen un papel primordial en el deporte de atletismo, en especial en las carreras de velocidad o sprinter.

La tarea principal de la Biomecánica deportiva consiste en análisis los movimientos deportivos; además de centrarse en la caracterización y optimización de los gestos técnicos, a partir de los movimientos dinámicos y cinemáticos de estos movimientos deportivos, de forma racional; así como de su perfeccionamiento técnico (Stuart, 2012, citado en Perdomo,2018, p. 26).

El estudio biomecánico de la técnica es de gran utilidad tanto para el entrenador como para el deportista, ya que les proporcionan información cuantitativa sobre el rendimiento, muchas veces se dan a conocer aspectos que no son apreciables hasta que se utilizan estudios biomecánicos.

El Programa Integral del Deportista (PID) en el evento de velocidad en el deporte de atletismo, constituye un documento, esencial desde la perspectiva metodológica que, orientada para los entrenadores deportivos, no se aprecia una caracterización del desplazamiento de los corredores, ni como realizar dicho análisis.

De lo anterior se desprende que la necesidad de un procedimiento biomecánico que permita el análisis de los primeros pasos de carrera de los velocistas juveniles de la Escuela de Iniciación Deportiva Escolar (EIDE) “Héctor Ruiz Pérez”, los principales objetivos son:

1. Establecer procedimientos biomecánicos para el análisis de los primeros pasos de carrera de los velocistas juveniles de la EIDE “Héctor Ruiz Pérez” de Villa Clara.

2. Conocer el nivel de satisfacción de los usuarios con el procedimiento propuesto, una vez aplicado con velocistas juveniles.

\section{MATERIALES Y MÉTODOS}

Se utilizaron métodos del nivel teóricos y empíricos que nos facilitaron la búsqueda de los resultados como: el inductivo-deductivo, el análisis y síntesis, el enfoque sistémicoestructural, el análisis de documentos, la entrevista, el estadístico-matemático, el estudio biomecánico, y el criterio de usuarios.

\section{Contexto y sujetos de la investigación.}

El contexto de estudio fue "Héctor Ruíz Pérez" de Villa Clara y el área donde habitualmente realizan la sesión de entrenamiento. 
Los sujetos investigados estuvieron: los cuatro atletas que conforman la población de velocistas de carreras planas, entre ellos tres masculinos y una femenina que pertenecen a la categoría juvenil, el entrenador de velocidad de la EIDE "Héctor Ruíz Pérez" de Villa Clara y el equipo de investigación Biomecánica de la Facultad de Cultura Física y Comisión Provincial de Atletismo.

Las variables de estudio se concretaron en los parámetros cinemáticos siguientes: grados de empuje en el despegue posterior, grados de amplitud en el paso anterior, grados de inclinación del tronco, longitud de pasos, velocidad, aceleración.

Criterio de usuarios relacionado con el sistema de indicadores fue empleado, de acuerdo con la Técnica de IADOV para evaluar la satisfacción de los usuarios, según los postulados teóricos (Toledo, et al., 2020).

Se empleó un cuestionario, el cual cuenta con un total de cinco preguntas cerradas y dos abiertas, cuya relación ignora el sujeto. La resultante de la relación de las 5 interrogantes cerradas nos permitió analizar la vinculación entre las preguntas, indicando la posición de cada sujeto en la escala de satisfacción, o sea su satisfacción individual.

Según Fernández y López (2014), esta técnica nos posibilitó alcanzar el índice de satisfacción grupal (ISG), para trabajar con los diferentes grados de satisfacción que se expresan en la escala numérica que oscila entre +1 y -1 de la siguiente forma:

Es necesario, hacer énfasis, 1 número resultante de la interrelación de las tres preguntas nos indica la posición de cada sujeto en la escala de satisfacción, o sea su satisfacción individual. La escala de satisfacción utilizada es la siguiente:

\section{Tabla 1.}

\section{Escala del índice de satisfacción grupal.}

\begin{tabular}{cl}
\hline Escala & \multicolumn{1}{c}{ Resultados } \\
\hline+1 & Máximo de satisfacción \\
0.5 & Más satisfecho que insatisfecho \\
0 & No definido y contradictorio \\
-0.5 & satisfecho \\
-1 & Máxima insatisfacción \\
\hline
\end{tabular}


La satisfacción grupal se calcula por la siguiente fórmula:

$$
\mathrm{ISG}=\frac{\mathrm{A}(+\mathbf{1})+\mathrm{B}(+\mathbf{0 . 5})+\mathrm{C}(0)+\mathrm{D}(-5)+\mathrm{E}(-1)}{\mathrm{N}}
$$

El índice grupal arroja valores entre $+1 \mathrm{y}-1$. Los valores que oscilan entre -1 y -0.5 . Indican insatisfacción; los comprendidos entre -0.49 y +0.49 evidencian contradicción y los que caen entre 0.5; 1 indican que existe satisfacción (Navas, 2011).

La técnica de IADOV contempla, además, dos interrogantes de carácter abierto López y González (2002), según los autores mencionados agregan dos interrogantes, que son importantes, estas son:

- ¿Qué importancia le concede al sistema de indicadores?

- ¿Cuáles son los aspectos que potencian o limitan el uso de este sistema de indicadores?

Estadístico: Se utilizó el paquete estadístico con el programa estadístico SPSS versión 23, nos permitió calcular la media, la mediana, la desviación estándar y coeficiente de variabilidad.

\section{RESULTADOS Y DISCUSIÓN}

Diagnóstico del estado actual del análisis de la técnica de las carreras de velocidad.

Al valorar los resultados de la entrevista a los entrenadores, miembros de la Comisión Técnica de Atletismo, se pudo constatar en el análisis de la técnica se realizó desde una visión empírica, pues siguen procederes tradicionales sin que se pongan de manifiesto la utilización de la biomecánica, ni el uso de la informática (Toledo, et al., 2020). Estos plantean que para resultar valioso el análisis de los gestos técnicos, debe cumplir con los procedimientos biomecánicos.

Criterios utilizados para la elaboración del procedimiento biomecánico para el análisis de los primeros pasos de carrera en corredores de velocidad

Para determinar los pasos para el análisis de los primeros pasos de carrera de los velocistas, se hizo un taller donde participaron profesores del colectivo de Biomecánica de la Facultad de Cultura Física "Marta Abreu" de Las Villas.

En el taller se dieron a conocer los objetivos de este y se presentó una primera versión de los pasos del proceder metodológico, los cuales son sometidos a discusión; 
posteriormente se llegó a consenso y se hizo la propuesta definitiva para el análisis de los primeros pasos de carrera en corredores de velocidad.

\section{Los procedimientos se encuentran de las siguientes formas.}

- Paso 1: filmación mediante las cámaras, situadas frontal y lateralmente a la acción del corredor.

- Paso 2: se digitalizó las imágenes de los fotogramas a fotogramas

- Paso 3: se sincronizó los datos obtenidos de cada cámara

- Paso 4: fase: obtención de resultados.

- Paso 5: procesamiento y análisis estadístico.

Puesta en práctica del procedimiento propuesto para el análisis de los primeros pasos de carrera en corredores de velocidad.

Una vez aplicado el procedimiento a los cuatro atletas de velocidad, se obtuvieron los siguientes datos:

La fase puesta en acción: esta explica de manera explícita, la parte de la carrera donde el corredor realiza el despegue de los tacos de salida, después de la reacción como respuesta del disparo ejecutada por el juez de salida; además el atleta trata de iniciar la carrera de manera eficiente y efectiva según sus gestos técnicos con la mayor velocidad que el corredor pueda alcanzar.

Con respecto a lo expresado, puede hallar que, en dos partes bien diferenciadas y consecutivas en el tiempo, como son: la reacción al disparo y el impulso del corredor sobre los tacos. De acuerdo con (Arufe y Martínez, 2005, citado en De la Torres, 2014, p. 15).

En relación con, De la Torre (2014), "enfatiza en dos factores, donde concedimos con el autor con relación a los 100 metros planos" (p.15). Podemos añadir que los factores frecuencia y longitud de los pasos están en correspondencia por una parte con el mayor o menor número de desplazamiento angular; así como por los segmentos que influyen del tren inferior, según los elementos del tiempo y la reacción al disparo y el impulso del corredor sobre los tacos de salida. 


\section{Tabla 2.}

Resultados de las variables, despegue posterior, paso anterior e inclinación del tronco, registrados en el 2018.

\begin{tabular}{cccc}
\hline Atletas & $\begin{array}{c}\text { Despegue } \\
\text { posterior }\end{array}$ & $\begin{array}{c}\text { Paso } \\
\text { anterior }\end{array}$ & $\begin{array}{c}\text { Inclinación } \\
\text { del tronco }\end{array}$ \\
\hline 1 & $168^{\circ}$ & $103^{\circ}$ & $85^{\circ}$ \\
2 & $162^{\circ}$ & $88^{\circ}$ & $46^{\circ}$ \\
3 & $161^{\circ}$ & $115^{\circ}$ & $57^{\circ}$ \\
4 & $156^{\circ}$ & $73^{\circ}$ & $51^{\circ}$ \\
Media & 161.75 & 94.75 & 59.75 \\
Mediana & 161.5 & 101.5 & 54 \\
Desviación & 3.25 & 14.25 & 12.63 \\
Coeficiente de Variabilidad & 0.02 & 0.14 & 0.23 \\
\hline
\end{tabular}

Podemos comprobar los resultados en la tabla dos, los cuatro velocistas juveniles de la EIDE, presentan diferentes ángulos de inclinación en los últimos cuatro pasos; es decir los datos se encuentran muy dispersos, lo cual se puede ver al valorar la desviación y el coeficiente de variabilidad, en el caso de la joven corredora que también es primer año de la categoría juvenil, muestra la menor inclinación con $85^{\circ}$, lo cual puede estar dado por mayor debilidad en músculos de la espalda y problemas en la técnica de carrera, el resto de los corredores presentan una aceptable inclinación destacándose, el corredor número dos. En sentido general, los valores de la mediana se comportan de manera satisfactoria para los diferentes ángulos, a pesar de lo antes señalado respecto a la atleta del sexo femenino.

Los valores del ángulo de despegue posterior y paso anterior determinan el largo de cada zancada y está a su vez está notablemente determinada por el largo de pierna y la fuerza producida en el menor tiempo.

Como ya se planteó, si tomamos como referencia las imágenes de la atleta uno, el ángulo de despegue posterior es mayor que los de los restantes atletas, al igual que el paso anterior, ya que la misma adopta una posición más erguida que los demás de manera prematura, o sea el tronco no está tan inclinado y por tanto el empuje de la pierna de despegue es más potente al no estar colocado muy por delante del centro de gravedad, 
además, las mujeres son más flexibles que los varones lo cual puede estar también influyendo.

\section{Figura 1.}

Ángulos del despegue posterior en los atletas juveniles
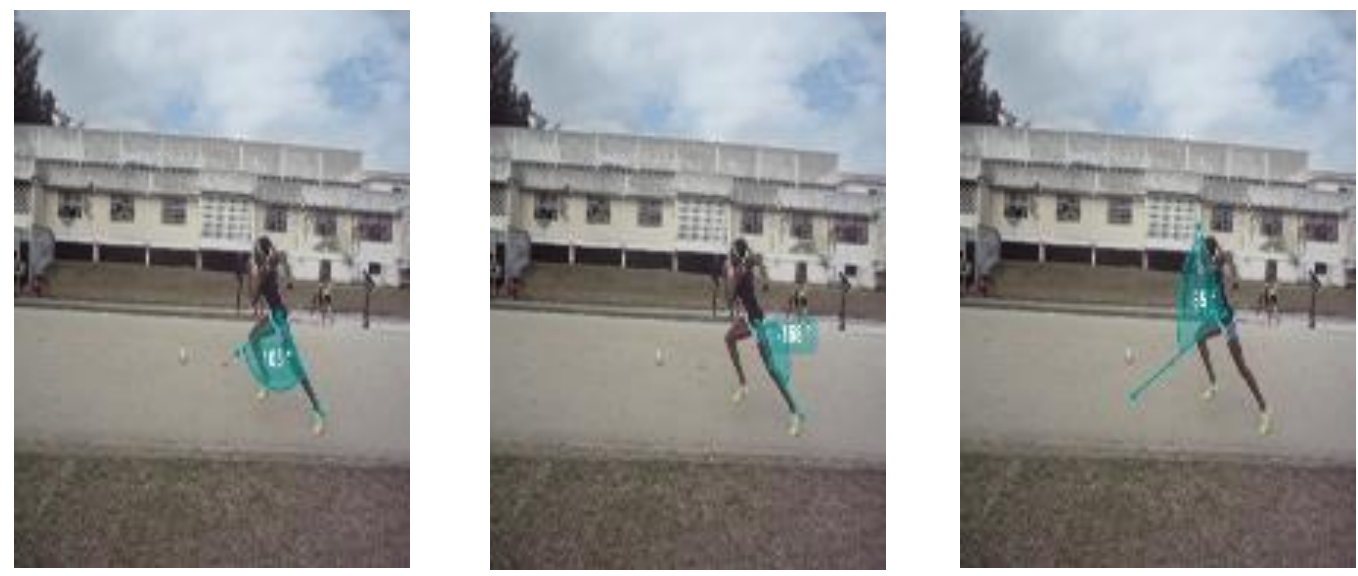

Al comparar la joven atleta con el atleta masculino número dos, se ve claramente la diferencia en los ángulos, sobre todo por el hecho de poseer una mejor técnica y mayores niveles de fuerza con relación a la joven diferentes ángulos de la técnica y niveles de fuerza.

\section{Figura 2.}

Ángulos del despegue posterior paso anterior e inclinación del tronco en los atletas juveniles.
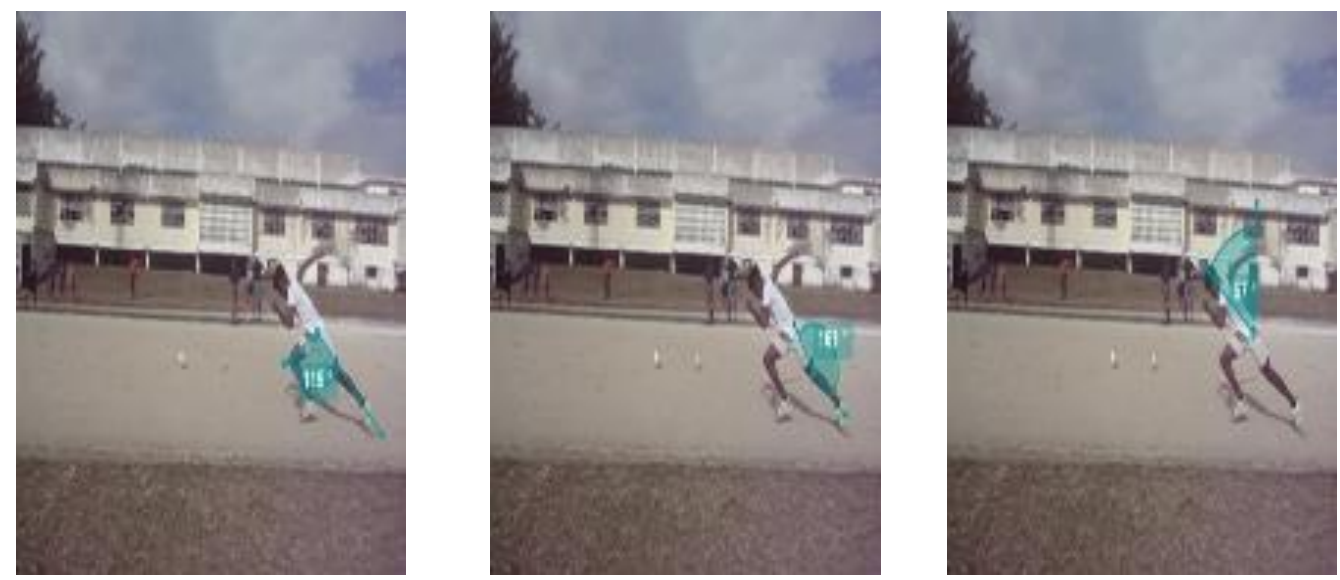


\section{Tabla 3.}

Resultados del comportamiento de la longitud en cada uno de los pasos en los atletas estudiados.

\begin{tabular}{ccccc}
\hline Atletas & Paso 1 & Paso 2 & Paso 3 & Paso 4 \\
\hline 1 & 1.37 & 2.04 & 2.20 & 2.26 \\
2 & 1.64 & 1.69 & 1.73 & 2.2 \\
3 & 1.76 & 1.77 & 1.77 & 2.01 \\
4 & 1.86 & 2.12 & 2.15 & 2.15 \\
Media & 1.66 & 1.91 & 1.96 & 2.16 \\
Mediana & 1.7 & 1.73 & 1.75 & 2.08 \\
Desviación & 0.15 & 0.18 & 0.21 & 0.07 \\
Coeficiente de Variabilidad & 0.09 & 0.10 & 0.12 & 0.04 \\
\hline
\end{tabular}

Según Vittori (1986, citado en De la Torre, 2014), el ritmo se puede definir como la sucesión ordenada de la forma de movimiento y la frecuencia con la que se repiten las diferentes fases.

Los factores frecuencia y longitud de los pasos están en correspondencia por una parte con el mayor o menor número de desplazamiento angular.

Por otro lado, es importante determinar una distribución de la amplitud de los pasos y el esfuerzo que optimice las posibilidades del atleta.

\section{La amplitud de los pasos en la fase de aceleración está determinada por:}

- Magnitud de fuerza.

- Impulso.

- Dirección de fuerza.

- Amplitud de movimiento aplicada.

También en esta fase es importante la dirección de fuerza, por lo que se considera importante:

- Relación del centro de masa con la base de apoyo.

- Aplicación de fuerza a línea de potencia.

- Ángulo de la espinilla.

- Secuencia de reclutamiento muscular.

- Extensores de caderas, luego extensores de rodilla. 
Como se aprecia en la tabla hay un incremento progresivo de la longitud del paso en todos los atletas, no obstante, el atleta tres mantiene la misma longitud en los pasos dos y tres y el atleta cuatro en los pasos tres y cuatro los atletas tres y cuatro.

Si se hace una valoración de los resultados de la mediana, podemos decir que hay un incremento progresivo lo cual favorece a la aceleración y la velocidad, sobre todo a la primera, manteniéndose los resultados bastante homogéneos por cada atleta en cada paso. Si comparamos los resultados de longitud de los pasos de atletas estudiados por GarcíaVerdugo (1972) oscilan en el cuarto paso entre 1.40 y 1.58 m que están por debajo de la mediana de los atletas estudiados, no obstante, consideramos que todavía estos atletas no tienen desarrollado los niveles óptimos de fuerza para lograr una mayor longitud.

Se hace notar que la longitud del paso de la atleta uno, que siendo de categoría inicial y del sexo femenino tiene la mayor longitud, pero es bueno aclarar que si es la de menor inclinación del tronco o sea que se yergue prematuramente, los pasos sean más largo, mientras que los atletas que poseen mayor inclinación, la longitud del paso sea menor que la atleta.

Es frecuente que los atletas, con menos experiencia existan una falta de progresión en la amplitud, en especial en el 2»y $3 »$ pasos.

Esta falta de progresión puede estar motivada por numerosas causas, pero fundamentalmente son dos:

a. Técnica. Un primer paso excesivamente largo con apoyo por delante del centro de gravedad provoca un frenazo y por consiguiente un segundo paso más corto.

b. De preparación. Poca fuerza en las piernas y como consecuencia se produce el hundimiento al no poder salir con prontitud de esta posición. Otros factores pueden ser faltos de coordinación, equilibrio, etc.

Gráfico 1. Atleta 1. Velocidad

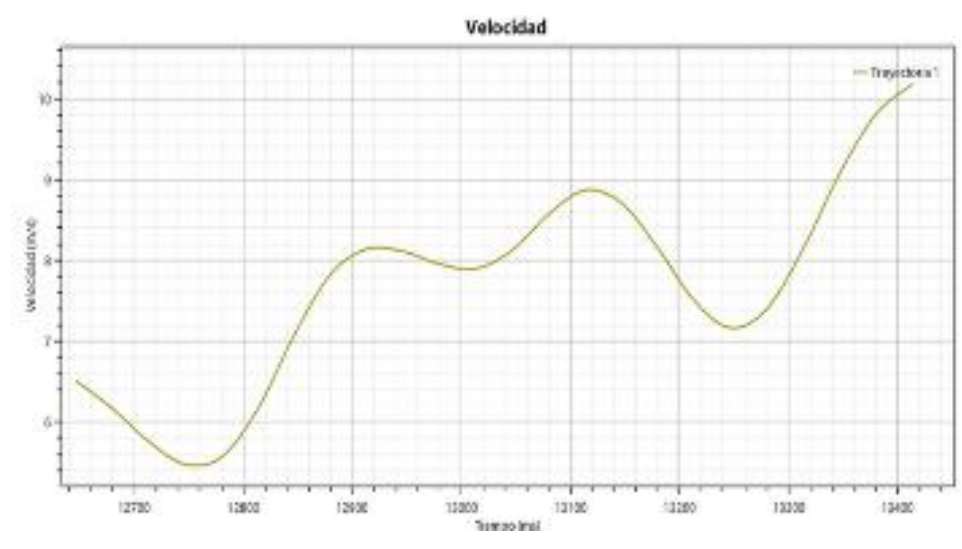


Como se puede apreciar la velocidad del atleta uno la cual hemos ya planteado se trata de una atleta y que es primer año de la categoría juvenil, presenta una velocidad ascendente en los 4 primeros pasos, pero con grandes variaciones que puede estar dado por modificaciones en los parámetros de los cuales depende la velocidad, que son la longitud (distancia que existe entre el apoyo anterior y despegue posterior, que a su vez depende de otros factores) y frecuencia de los pasos (número de pasos en la menor unidad de tiempo) y por tanto las modificaciones que existan entre ellos provocarán oscilaciones en la misma. (García-Verdugo, 1972).

\section{Gráfico 2. Atleta 1. Aceleración}

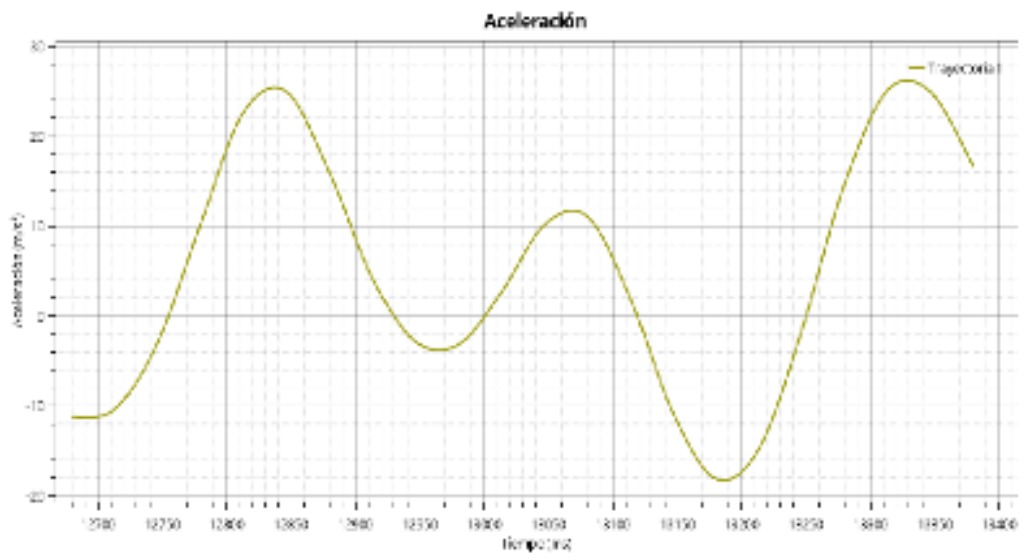

La aceleración es un cambio positivo en la velocidad dentro de un determinado intervalo de tiempo. La aceleración es proporcional a la fuerza que la provoca.

Fuerza $(F)=$ masa $(\mathrm{m}) \times$ aceleración $(\mathrm{a})$

\section{Aceleración pura:}

- Máximo índice de aceleración

- Fuerza generada en gran medida por la contracción muscular.

- $75-80 \%$ de máxima, sostenida.

Esta fase de aceleración rápida se caracteriza por la progresión muy acentuada que sufre la velocidad al aumentar la frecuencia y longitud de los pasos, ambos parámetros aumentan de una forma muy rápida y progresiva, y como consecuencia la velocidad aumenta de esa manera también. (García-Verdugo, 1972).

Es así como, al momento de iniciar el disparo hasta los 20 m, la aceleración es máxima y a partir de ese punto, dicha aceleración se hace menor de 1 metro/segundo.

Como se puede apreciar la velocidad es ascendentes en los 4 primeros pasos, pero con grandes variaciones como ocurre con la velocidad, provocado también por las 
modificaciones de los parámetros de los cuales depende la velocidad, que a su vez se manifiesta en varios factores por la fuerza de los estímulos nerviosos, la fuerza, la técnica de carrera entre otros.

En el caso de esta atleta, la variabilidad de la aceleración se hace notable y puede estar dado por tener menos niveles de fuerza que los varones y una técnica aún no perfeccionada.

\section{Gráfico 3. Atleta 2. Velocidad}

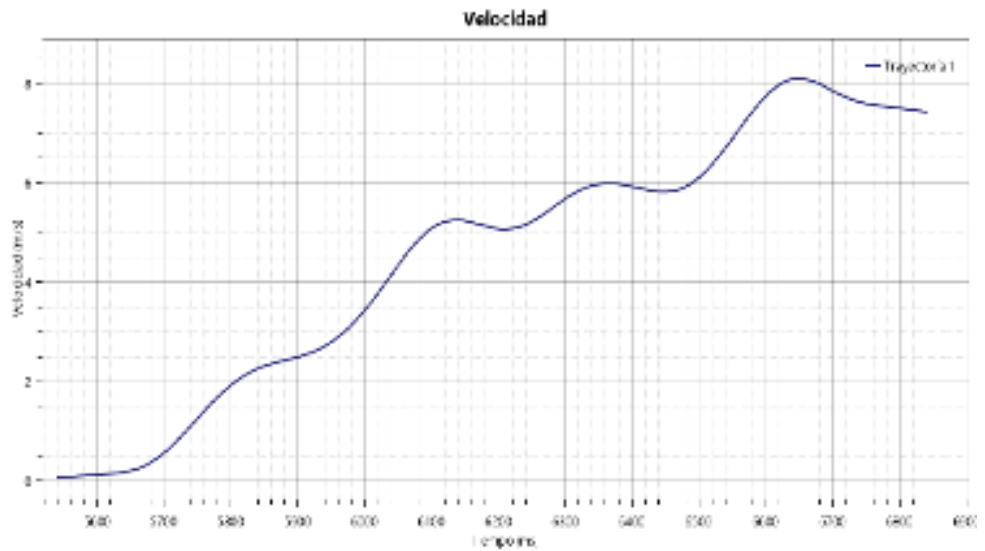

Como se puede apreciar la velocidad del atleta es ascendentes en los 4 primeros pasos, pero y las variaciones se pueden catalogar de mínimas dado por no grandes modificaciones en los parámetros de los cuales depende la velocidad, que son la longitud y frecuencia de los pasos y por tanto las oscilaciones en la misma es menor. (GarcíaVerdugo, 1972).

\section{Gráfico 4. Atleta 2. Aceleración}

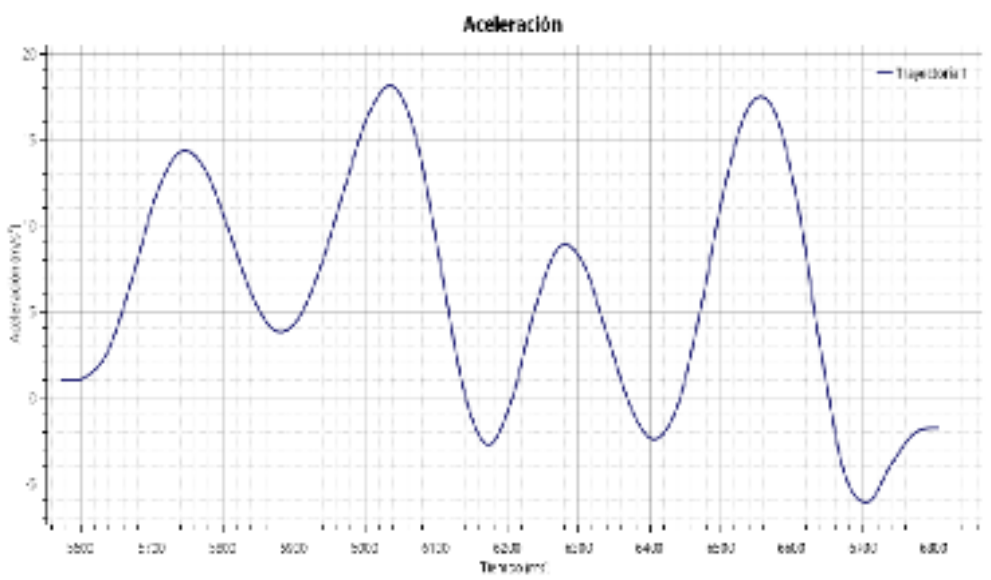

En el caso de la aceleración se comporta con grandes oscilaciones lo cual es típico de la aceleración, producto de que estos pasos necesariamente deben ser coordinados y con un 
ritmo donde se convino de manera eficiente la frecuencia y longitud de paso, así como el tiempo de vuelo.

\section{Gráfico 5. Atleta 3. Velocidad}

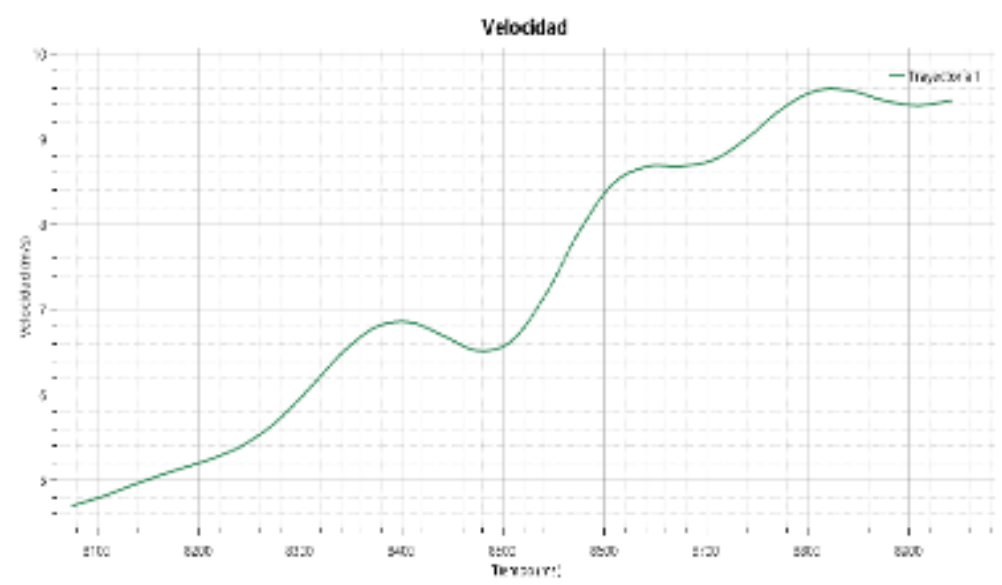

Como se puede apreciar la velocidad del atleta tres es ascendentes en los 4 primeros pasos, pero y las variaciones son notables respecto al atleta número dos, producto de que igual que la atleta uno puede existir grandes modificaciones en los parámetros de los cuales depende la velocidad, que son la longitud y frecuencia de los pasos y por tanto las oscilaciones en la misma es menor. (García-Verdugo, 1972).

Gráfico 6. Atleta 3. Aceleración

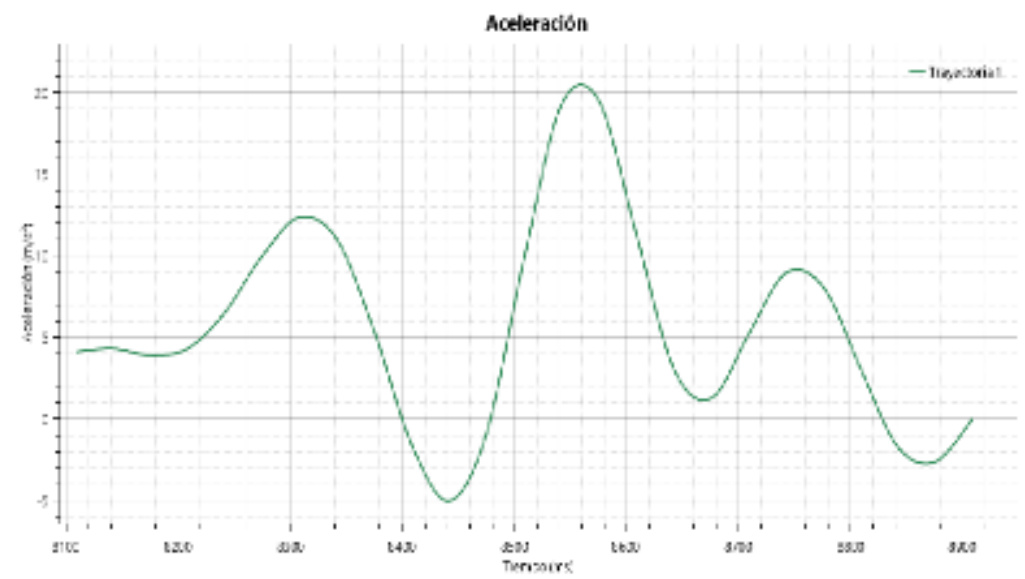

La aceleración se ha comportado como los atletas anteriores y se puede decir que es un parámetro bien difícil de regular pues hay que incrementar progresivamente la longitud y frecuencia de pasos para lo cual hay que tener un ritmo riguroso, no obstante, es un elemento importante para valorar por el entrenador. 
Gráfico 7. Atleta 4. Velocidad

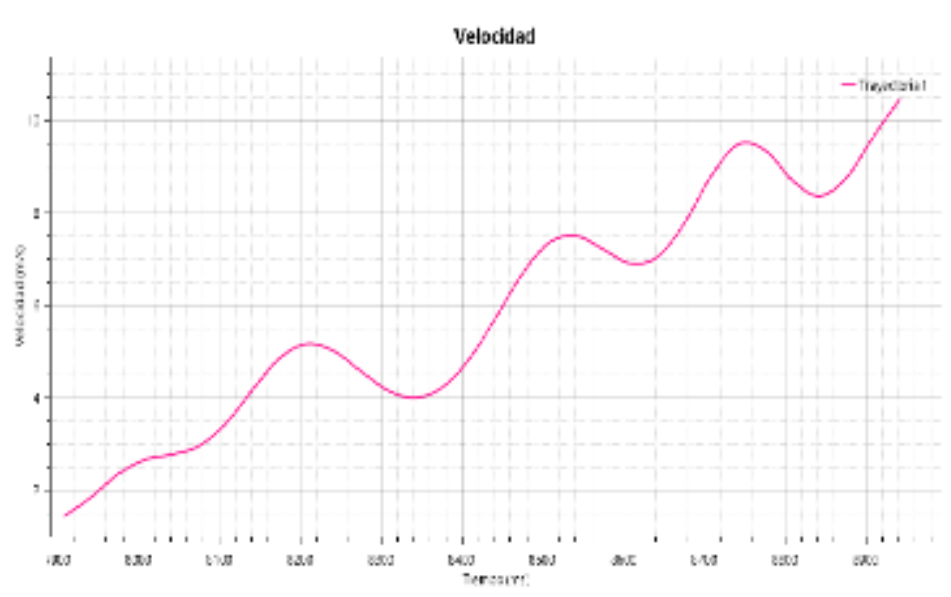

Gráfico 8. Atleta 4. Aceleración

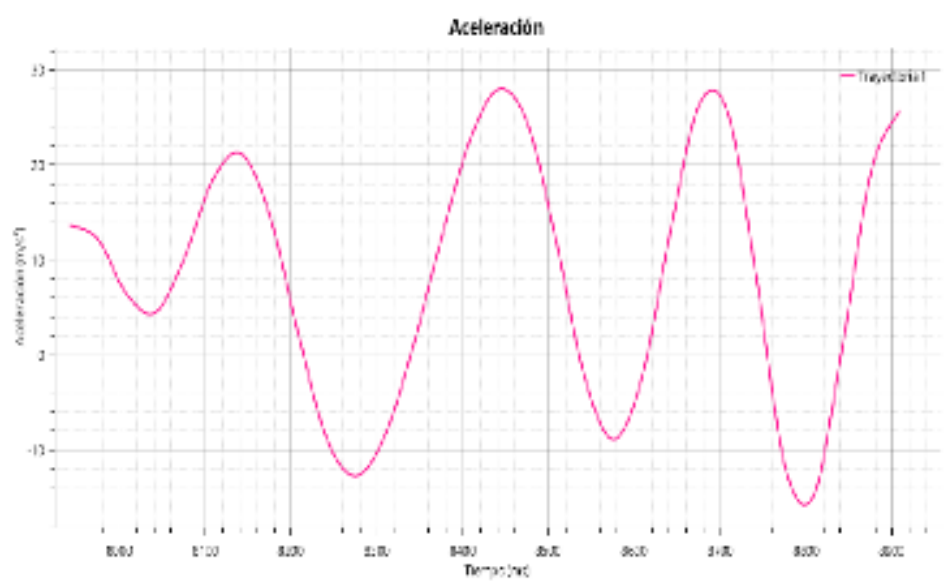

La velocidad del atleta tres es ascendentes en los 4 primeros pasos, pero las variaciones son notables respecto al atleta número dos y tres, producto de que igual que la atleta uno puede existir grandes modificaciones en los parámetros de los cuales depende la velocidad, que son la longitud y frecuencia de los pasos y por tanto las oscilaciones en la misma es menor. (García-Verdugo, 1972).

La aceleración es similar en su comportamiento a los atletas anteriores, por lo que de manea general se puede decir que a pesar de que son atletas juveniles y de alto rendimiento, estos deben continuar trabajando el ritmo de estos pasos de carrera, estrategia de carrera, en el incremento de la fuerza y en la mejora de la técnica.

Resultados del criterio de los usuarios introductores sobre la propuesta procedimiento biomecánico para el análisis de la variabilidad de la ejecución técnica de los primeros pasos de carrera de los velocistas juveniles 


\section{Valoración de la propuesta por los usuarios}

Según los resultados obtenidos con relación al índice de satisfacción grupal (ISG), dedicado a los usuarios introductores.

Estos usuarios están conformados por 4 miembros de la Comisión Técnica de Atletismo y el entrenador principal de Velocidad que a su vez funge como jefe del colectivo técnico de Atletismo.

\section{La satisfacción grupal se calcula a través de la siguiente fórmula:}

ISG $=\mathrm{A}(+1)+\mathrm{B}(+0.5)+\mathrm{C}(0)+\mathrm{D}(-5)+\mathrm{E}(-1) / \mathrm{N}$

El índice alcanzado indica que existe satisfacción de los usuarios introductores con el procedimiento propuesto, a pesar de que hacen varias reflexiones al contestar las preguntas abiertas, que son tenidas en cuenta en su perfeccionamiento.

Las respuestas a estas preguntas están asociadas a la necesidad de implementar formas de superación que permitan un rápido adiestramiento con los pasos determinados para el uso de la biomecánica en el análisis de los primeros pasos de carrera.

El Programa Integral de Preparación del Deportista de las carreras planas y con vallas, se comprobó que los procedimientos para la técnica juegan un papel esencial en el desarrollo de los ángulos despegue posterior, paso anterior e inclinación del tronco.

Sin embargo, estos no hacen menciones a los entrenadores, del análisis biomecánico de la técnica y sus procedimientos para cumplimentar la correcta explicación e interpretación y compresión de los movimientos técnicos desde la ciencia biomecánica.

$\mathrm{Al}$ respecto para entender la naturaleza de los problemas biomecánicos, muchas variables y términos han sido utilizados para tratar de explicar el fenómeno que se manifiestan para el análisis de los primeros pasos de carrera de los velocistas (Knudson, 2007).

El desarrollo de tecnología y su relación con la biomecánica se suma a la precisión de las mediciones para un mejor análisis de los factores claves para el rendimiento de los velocistas o sprint. La Aceleración de arranque y bloqueo de sprint son los dos primeros derivados de la velocidad de sprint donde el atleta intenta asumir la velocidad máxima de bloqueo (Čoh, et at., 2006, p.104).

La técnica constituye un aspecto determinante en el proceso formación deportiva de los atletas de velocidad, durante su proceso la corrección de errores de estos gestos técnicos es importantes en los atletas principiantes, es donde los entrenadores deben tener presente los aspectos pedagógicos, didácticos y metodológico del deporte (Pérez et al., 2020). 
"La técnica deportiva el factor determinante del rendimiento que engloba el conjunto de movimientos racionales, que conducen al deportista de manera funcional, económica y adaptada a la obtención de óptimos resultados deportivos" (Álvarez, 2014, p.4).

La ciencia y la técnica se han incorporado al mundo del deporte con el fin de explicar fenómenos y técnicas con el fin de buscar la mejora del rendimiento y explicar los fenómenos ligados a la motricidad humana. La biomecánica ocupa un factor determinante entre las ciencias que conforma la actividad física y el deporte (Padullés, 1992).

Procedimiento para el análisis de los primeros pasos de carrera de los velocistas, estas acciones se suelen producir en todos los velocistas indistintamente de su nivel, es en la duración y calidad de estas donde se pueden observar las diferencias entre atletas (Floría, \& Ferro, 2011, p.409).

\section{CONCLUSIÓN}

De acuerdo con los resultados obtenidos, según el estado actual arribamos a la conclusión que se necesita el empleo de la tecnología para la elaboración de procedimientos biomecánicos para los usuarios que expresan una alta satisfacción para el análisis de la técnica de las carreras de velocidad plana que se constató con el uso del programa Kinovea.

\section{REFERENCIAS BIBLIOGRAFICAS}

Álvarez, D. (2014). Valoración biomecánica de la carrera de velocidad. [Trabajo en Ciencias de la Actividad Física y del Deporte]. Universidad de León. https://buleria.unileon.es/bitstream/handle/10612/4170/1_TFG\%20David\%20\% C3\%81lvarez\%20Romero.pdf?sequence $=1$

Arufe, V., y Martínez, M. J. (2005). Tratado de atletismo en el siglo XXI. Asociación Cultural. Atlética Gallega.

Bezodis, N. E. (2009). Biomechanical investigations of sprint start technique and performance. [A Doctoral Thesis of Philosophy]. University of Bath. https://researchportal.bath.ac.uk/en/studentTheses/biomechanical-investigationsof-sprint-start-technique-and-perfor

Burruel, J. A., Castro, A. I., López, L., y Gallardo, C. I. (2016). Análisis cinemático de la técnica de salida en 100 metros planos en corredores universitarios. Revista EFDeportes, 21(216), 1-1. 
https://www.efdeportes.com/efd216/analisis-cinematico-en-100-metrosplanos.htm

Campistrous, L., y Rizo, C. (2006). El criterio de expertos como método en la investigación educativa. Editorial. Instituto Superior de Cultura Física "Manuel Fajardo".

Carrillo, C. (2014). Análisis cinético y cinemático de la carrera de velocidad 100 metros lisos en sus diferentes fases. [Trabajo para la obtención del Título de Graduado en Ciencias del Deporte]. Universidad Politécnica de Madrid. https://oa.upm.es/31039/1/TFG_CESAR_CARRILLO_CORDON.pdf

Čoh, M., Peharec, S., Bačić. P., \& Mackala, K. (2017). Biomechanical Differences in the Sprint Start Between Faster and Slower High-Level Sprinters. Journal of Human Kinetics, (56), 29-38. doi: 10.1515/hukin-2017-0020 https://pubmed.ncbi.nlm.nih.gov/28469741/

Čoh, M., Tomažin, K., \& Štuhec, S. (2006). The biomechanical model of the sprint starts and block acceleration. Physical Education and Sport, 4 (2),103-114. https://elitetrack.com/article_files/biomechanics-block-start.pdf

Čoh, M., \&Tomažin, K. (2006). Kinematic analysis of the sprint starts and acceleration from the blocks. New Studies in Athletics, 21(3), 23-33. http://centrostudilombardia.com/wp-content/uploads/IAAF-Corsa-Velocita/2006 Kinematic-analysis-of-the-sprint-start.pdf

Comín, M., Dejoz, R., Peris, J. L. C., Atienza, C., y Vera, P. (1996). Conceptos básicos de mecánica en biomecánica. Revista Biomecánica, lV, (7), 96-109. http://hdl.handle.net/2099/5496

De la Torre, G. (2014). ¿Puede ser la estrategia de carrera un factor de optimización del rendimiento en los 100 metros lisos?. [Tesis en Ciencias de la Actividad Física y del Deporte]. Universidad Politécnica de Madrid. https://oa.upm.es/31068/1/TFG_GERMAN_DE_LA_TORRE_ORELLANA.pdf

Estrada, Y. C. (2018). Biomecánica: de la física mecánica al análisis de gestos deportivos. Ediciones. USTA. Universidad Santo Tomás. https://repository.usta.edu.co/bitstream/handle/11634/12464/Obracompleta.2018 Estradayisel.pdf?sequence $=1 \&$ isAllowed $=\mathrm{y}$ 
Fernández, A. y López, A. (2014). Validación mediante criterio de usuarios del sistema de indicadores para prever, diseñar y medir el impacto en los proyectos de investigación del sector agropecuario. Revista Ciencias Técnicas Agropecuarias, 23(3), 77-82. http://scielo.sld.cu/scielo.php?script=sci_arttext\&pid=S2071$\underline{00542014000300012 \& \operatorname{lng}=\mathrm{es} \& \operatorname{tlng}=\mathrm{es}}$

Floría, P., y Ferro, A. (2011). Análisis del rendimiento en competición entre corredores de Análisis del rendimiento en competición entre corredores de 100 metros lisos de diferente nivel 100 metros lisos de diferente nivel. Revista Internacional de Ciencias del Deporte, VII (VII), 408-416. doi:10.5232/ricyde2011.02606 https://www.redalyc.org/articulo.oa?id=710/71020064007

García, T. (2019). Cinemática de la técnica de carrera en atletas con diversidad funcional visual: Un análisis comparativo. [Trabajo de fin de Grado]. Universidad de Sevilla. https://hdl.handle.net/11441/91099

García-Verdugo (1972). Análisis de los 100 metros lisos. Velocidad-frecuencia, consideraciones técnicas. Instituto Nacional de Educación Física y Deportes. https://oa.upm.es/13059/

Knudson, D. \& Morrison, C. (2002). Qualitative analysis of human movement (2nd edn.) Champaign: Human Kinetics.

Knudson, D. (2007). Qualitative biomechanical principles for application in coaching. Sports biomechanics. International Society of Biomechanics in Sports, 6

109-18.

doi: 10.1080/14763140601062567https://www.researchgate.net/publication/629452

López, A. y González, V. (2002). La técnica de IADOV. Una aplicación para el estudio de la satisfacción de los alumnos por las clases de educación física. Revista EFDeportes, 8 (47), 1-1. https://www.efdeportes.com/efd47/iadov.htm

Mackala, K. (2007). Optimisation of performance through kinematic analysis of the different phases of the 100 metres. Sprint Magazine, 22 (2), 7-16. https://www.iat.uni-leipzig.de/datenbanken/iks/sponet/Record/3041256

Muñiz, A. (2018). Los 100 metros planos. La especialización temprana y la formación de atletas élite. Editorial. Universidad Iberoamericana.

Navas, A. E. (2011). Propuestas de actividades recreativas para potenciar la cultura ambientalista. Editorial. Monografia. 
https://www.monografias.com/trabajos87/actividades-recreativas-culturaambientalista/actividades-recreativas-cultura-ambientalista3.shtml

Perdomo, J. M. (2018). La superación profesional de los entrenadores deportivos de villa clara en la aplicación de la biomecánica. [Tesis presentada en opción al grado científico de Doctor en Ciencias Pedagógicas]. Universidad Central "Marta Abreu" de las Villas.

https://dspace.uclv.edu.cu/bitstream/handle/123456789/11252/Juan\%20M.Perdo $\underline{\text { mo\%20-Tesis\%20Defensa.\%20OK.pdf?sequence }=1 \& \text { isAllowed }=y}$

Pedullés, J. M. (1992). Biomecánica del deporte. Enciclopedia Océano,1, 1-22. Universidad de Barcelona.

https://www.academia.edu/21765498/Biomec\%C3\%A1nica_del_Deporte_JM_P adull\%C3\%A9s

Pérez, A. J., Gutiérrez. A. E., Sarabia, H. M., Vega, S. I., y Bernal, F. (2020). Proceso de corrección en errores técnicos para los atletas en formación. (Ponencia). VIII Congreso Internacional "Desafíos de la actividad física y el deporte durante la pandemia COVID 19” UNISON, Sonora, México.

http://www.congresosdeportes.uson.mx/docs/MEMORIACONGRESO2020.pdf

Rius, J., Padullés, J. M., López, J. L., Sánchez, R., Rosich, M., Sánchez, L., y Escalona, M. (2006). Metodología y técnicas de atletismo. Editorial. Paidotribol.

Stuart, W. (2012). Biomecánica aplicada al deporte: contribuciones, perspectivas y desafíos. Revista EFDeportes, 17 (170), 1-10.

https://www.efdeportes.com/efd170/biomecanica-aplicada-al-deporte.htm

Toledo, R., Medina, M. L., Rodríguez, J. R., Denis, C., y Sánchez, A. B. (2020). Procedure for the analysis of the biomechanics of the variability of the movement in the discus throwing. Revista de Ciencia y Tecnología en la Cultura Física, 15(3), 518-533.http://scielo.sld.cu/scielo.php?script=sci_arttext\&pid=S199624522020000300518\&lng=es\&tlng=en

Vittori, C. (1986). Ipotesi di un modello ritmico della corsa dei $100 \mathrm{~m}$. e sua utilizzazione come metodo di controllo dell'allenamento. Reviste Atletica Studi. (6), 451-456. http://centrostudi.fidal.it/centrostudi/web/index.php?r=item\%2Fview\&id=377 